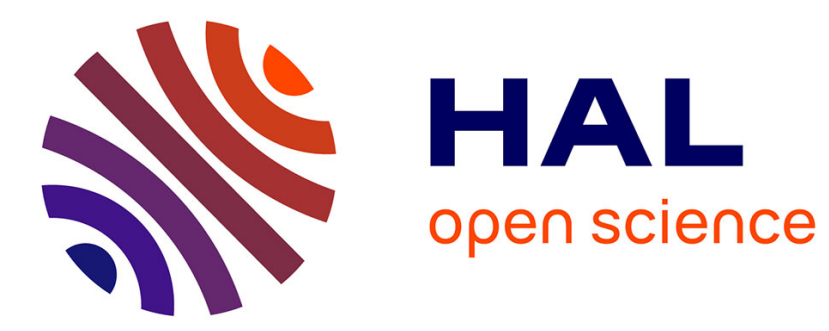

\title{
Étude de la structure de multicouches Au/Pt par des techniques complémentaires de rayons $\mathrm{X}$
}

\author{
L. Bimbault, K. Badawi, P. Goudeau, J. Mimault, O. Proux
}

\section{To cite this version:}

L. Bimbault, K. Badawi, P. Goudeau, J. Mimault, O. Proux. Étude de la structure de multicouches $\mathrm{Au} / \mathrm{Pt}$ par des techniques complémentaires de rayons X. Journal de Physique IV Proceedings, 1996, 06 (C7), pp.C7-43-C7-51. 10.1051/jp4:1996706 . jpa-00254494

\section{HAL Id: jpa-00254494 https://hal.science/jpa-00254494}

Submitted on 1 Jan 1996

HAL is a multi-disciplinary open access archive for the deposit and dissemination of scientific research documents, whether they are published or not. The documents may come from teaching and research institutions in France or abroad, or from public or private research centers.
L'archive ouverte pluridisciplinaire HAL, est destinée au dépôt et à la diffusion de documents scientifiques de niveau recherche, publiés ou non, émanant des établissements d'enseignement et de recherche français ou étrangers, des laboratoires publics ou privés. 
JOURNAL DE PHYSIQUE IV

Colloque C7, supplément au Journal de Physique III, Volume 6, novembre 1996

\title{
Étude de la structure de multicouches Au/Pt par des techniques complémentaires de rayons $X$
}

\author{
L. Bimbault, K.F. Badawi, Ph. Goudeau, J. Mimault et O. Proux \\ Laboratoire de Métallurgie Physique, URA 131 du CNRS, Université de Poitiers, UFR SFA, SP2MI, \\ Boulevard 3, Téléport 2, BP. 179, 86960 Futuroscope cedex, France
}

\begin{abstract}
Résumé : Des multicouches $\mathrm{Au} / \mathrm{Pt}$ de faible période ont été étudiées par diffraction des rayons $\mathrm{X}$ et par spectrométrie d'absorption X. Les spectres de diffraction mesurés pour tous les échantillons présentent un seul système de raies caractéristiques d'une structure cubique face centrée dont le paramètre de maille serait compris entre ceux de l'or et du platine massifs. L'influence de la périodicité est clairement mise en évidence sur les spectres de diffraction $\mathrm{X}$ aux petits et grands angles. Les résultats de diffraction des rayons $\mathrm{X}$ sont en désaccord avec les résultats de spectrométrie d'absorption $X$. Cette contradiction entre les résultats apportés par ces deuX techniques souligne l'intérêt de les conjuguer pour étudier des systèmes multicouches de très faibles périodes.
\end{abstract}

\section{Introduction}

Au niveau fondamental, les structures multicouches offrent une opportunité unique de mise en évidence des effets d'interface sur l'état mécanique et microstructural de matériaux biphasés. Ces effets ont une importance d'autant plus grande que la période de la multicouche est faible. Les contraintes et la microstructure sur d'autres multicouches, ayant des périodicités de l'ordre de dix nanomètres, ont été étudiées au Laboratoire de Métallurgie Physique (Poitiers) au cours de précédents travaux $[1,2,3]$.

Dans ce travail, nous avons choisi d'étudier le système $\mathrm{Au} / \mathrm{Pt}$ de composition équiatomique pour de faible période $(2,4 \mathrm{~nm}$ et $5,6 \mathrm{~nm}$ par bicouche) par diffraction et par spectrométrie d'absorption des rayons X. Les éléments Au et $\mathrm{Pt}$ pour cette composition et pour les conditions de température $\left(\approx 300^{\circ} \mathrm{K}\right)$ du dépôt sont immiscibles, condition nécessaire à la non diffusion des éléments aux interfaces. Ces éléments sont facilement observables par diffraction des rayons $\mathrm{X}$ car leurs facteurs de diffusion atomique sont élevés et leurs structures cristallographiques sont identiques ( $\mathrm{cfc}$ ) avec une différence de paramètre de maille de l'ordre de $4 \%$.

Un article récent [4] a montré que la diffraction des rayons $\mathrm{X}$ ne permettait pas dans le cas des très faibles périodes sur le système cuivre-cobalt de distinguer entre un mélange $A B$ et une multicouche $A / B$. Dans cette étude, nous montrons que ces conclusions s'appliquent aussi au système or-platine. C'est pourquoi il est intéressant d'associer la spectrométrie d'absorption des rayons $X$ à la diffraction des rayons $X$ pour l'étude de nos multicouches faites par évaporation au canon à électron où l'irrégularité des couches est grande du fait du mode de croissance en "cluster" de l'or et du platine. 


\section{Expérimentation}

\subsection{Condition de dépôt}

Nous avons déposé sur des substrats de silicium monocristallins orientés (100), d'épaisseur $200 \mu \mathrm{m}$, préalablement oxydée en surface (diffraction des rayons X et courbure) et de silice fondue d'épaisseur $2 \mathrm{~mm}$ (EXAFS), quatre séries différentes d'échantillons par évaporation au canon à électron sous un vide poussé $\left(10^{-7}\right.$ torr) et à température ambiante $(\approx 300 \mathrm{~K})$. Les épaisseurs de chaque strate sont contrôlées au cours du dépôt par un cristal piézo-électrique fixé parallèlement aux échantillons. Ces quatre séries se distinguent les unes des autres par la permutation des valeurs de deux paramètres qui sont la nature de la première couche déposée et l'épaisseur de la période. Les différents paramètres de dépôts sont récapitulés par série dans le tableau 1 :

\begin{tabular}{|c|c|c|c|c|c|c|c|}
\hline $\begin{array}{c}\text { série } \\
\mathrm{n}^{\circ}\end{array}$ & $\begin{array}{c}\text { nature de la } \\
1^{\circ} \text { couche }\end{array}$ & $\begin{array}{c}\text { période } \\
(\mathrm{nm})\end{array}$ & $\begin{array}{c}\text { nombre de } \\
\text { bicouches }\end{array}$ & $\begin{array}{c}\text { épaisseur } \\
\text { totale }(\mathrm{nm})\end{array}$ & $\begin{array}{c}\text { vitesse de } \\
\text { dépôt }\left(\mathrm{nm} \mathrm{s}^{-1}\right)\end{array}$ & $\begin{array}{c}\text { température } \\
\text { de dépôt }(\mathrm{K})\end{array}$ & $\begin{array}{c}\text { vide } \\
\text { torr }\end{array}$ \\
\hline 1 & $\mathrm{Au}$ & 2,4 & 42 & 100,8 & 0,1 & 300 & $10^{-7}$ \\
\hline 2 & $\mathrm{Pt}$ & 2,4 & 42 & 100,8 & 0,1 & 300 & $10^{-7}$ \\
\hline 3 & $\mathrm{Au}$ & 5,6 & 18 & 100,8 & 0,1 & 300 & $10^{-7}$ \\
\hline 4 & $\mathrm{Pt}$ & 5,6 & 18 & 100,8 & 0,1 & 300 & $10^{-7}$ \\
\hline
\end{tabular}

Tableau 1 : conditions de dépôt

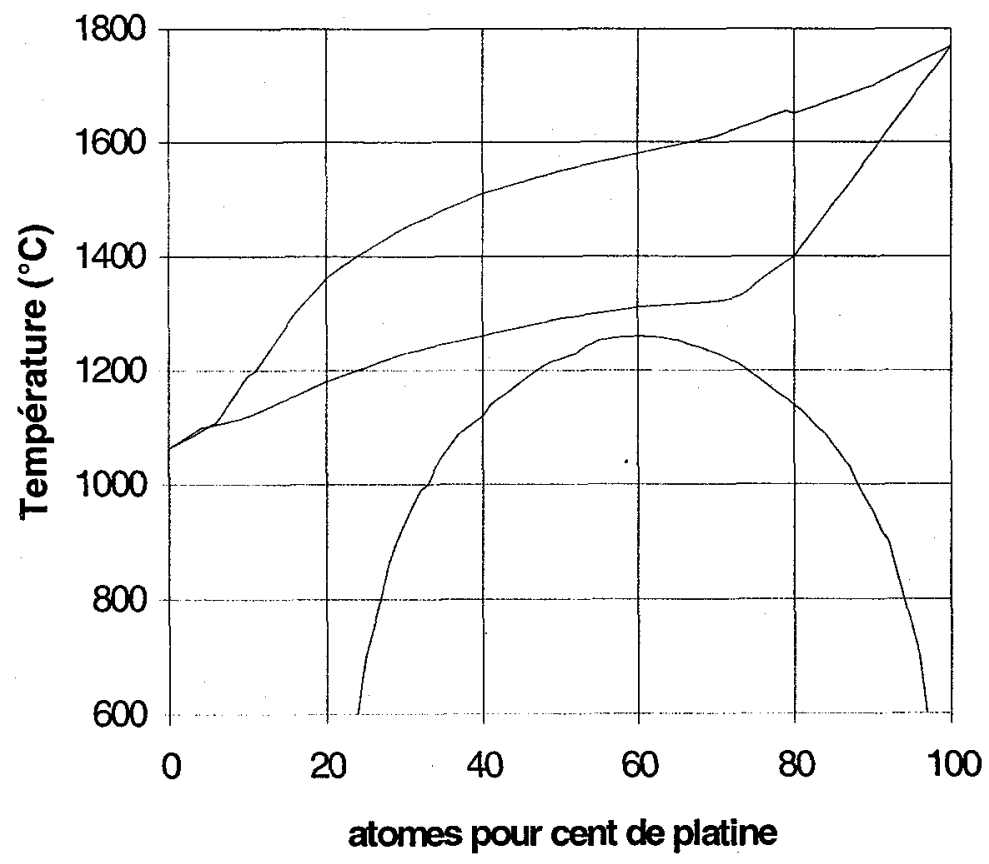

Figure 1 : diagramme de phase Au-Pt 
Nous donnons, figure 1, le diagramme de phase or-platine [5]. Il exclut à l'équilibre et à l'ambiante tout mélange équiatomique de ces deux éléments. Selon ce diagramme, un éventuel mélange de ceux-ci ne peut être dû qu'à la méthode de dépôt. Entre l'évaporation d'une monocouche et de la suivante, il existe un temps de latence qui exclut toute coévaporation intempestive. Les énergies de dépôt par évaporation au canon à électron sont très faibles, et ceci devrait exclure tout phénomène de mélange. La seule possibilité de mélange pour ces deux éléments est une interdiffusion aux interfaces après dépôt. Une telle hypothèse est peu probable à température ambiante compte tenu du coefficient d'interdiffusion du platine dans l'or. Les valeurs relevées dans les tables [6] pour ce coefficient donnent à température ambiante :

$$
\begin{aligned}
& \mathrm{D}=\mathrm{D}_{0} \times \mathrm{e}^{-\mathrm{E} / \mathrm{RT}} \\
& \mathrm{D}=1,24 \times 10^{-3} \times \mathrm{e}^{-39000 /(1,986 \times 300)} \\
& \mathrm{D}=4,7 \times 10^{-32} \mathrm{~cm}^{2} / \mathrm{s} .
\end{aligned}
$$

\subsection{Méthodes de caractérisation}

\subsubsection{Diffraction des rayons $\mathrm{X}$.}

Les expériences de diffraction X ont été effectués d'une part sur la ligne D22 au LURE (Orsay-France) sur l'anneau D.C.I. et d'autre part sur un diffractomètre 4-cercles construit dans notre laboratoire (LMP Poitiers) installé sur une source à anode tournante type RIGAKU [7].

2.2.1.1 La méthode des $\sin ^{2} \psi$ : contraintes résiduelles et paramètre libre de contraintes.
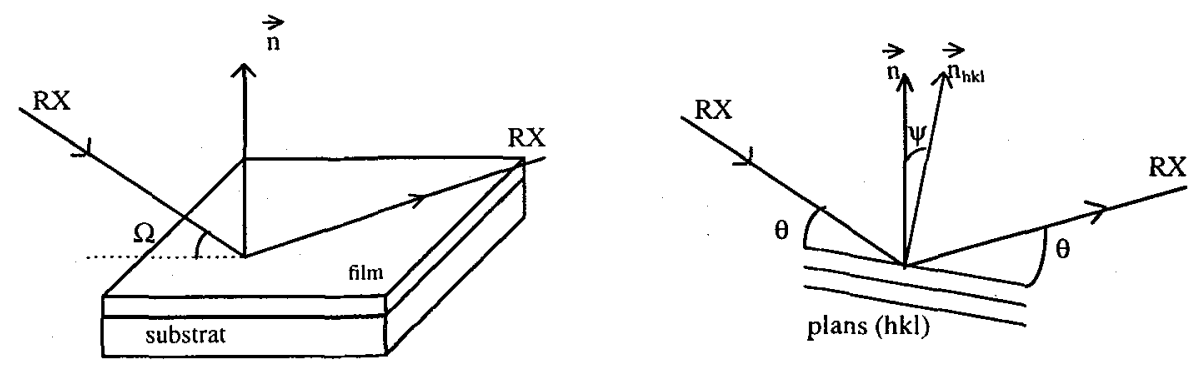

Figure 2: Définition des angles de mesures. $\Omega=\theta-\psi$, $\psi$ étant l'angle entre la normale à la surface et celle aux plans diffractants

Son principe est simple : il consiste à mesurer l'angle $2 \theta$ de diffraction d'une famille donnée de plans $\{\mathrm{hkl}\}$ pour différentes inclinaisons $\psi$ (soit différentes orientations des grains diffractants). Si on fait l'hypothèse que les contraintes sont biaxiales et isotropes dans le plan de surface par rapport à la surface du film (figure 2); les angles $\theta$ de Bragg des pics sont reliés à la contrainte $\sigma$ par la relation suivante :

$$
\ln (1 / \sin \theta)=1 / 2 S_{2} \sigma \sin ^{2} \psi+2 S_{1} \sigma+\ln \left(1 / \sin \theta_{0}\right)
$$

Où $1 / 2 S_{2}$ et $S_{1}$ sont les constantes élastiques radiocristallographiques (CER) du matériau et $\theta_{0}$ l'angle de Bragg correspondant au paramètre de maille libre des contraintes. En traçant 
$\ln (1 / \sin \theta)$ en fonction de $\sin ^{2} \psi$, on obtient une droite dont la pente donne la contrainte et l'ordonnée à l'origine $\theta_{0}$ soit le paramètre de maille libre de contraintes.

La diffraction aux grands angles des rayons $\mathrm{X}$ ne permet de chiffrer que les contraintes dans les domaines cristallisés. En complémentarité, les contraintes globales $\sigma^{*}$ sont mesurées par profilométrie. Ces dernières sont reliées au rayon de courbure $\mathrm{R}$ du substrat par la formule de Stoney [8]:

$$
\sigma=E^{*}\left(H^{2} / 6 h\right)(1 / R)
$$

où $\mathrm{E}^{*}$ est le bimodule du substrat, $\mathrm{h}$ l'épaisseur totale de la multicouche et $\mathrm{H}$ celle du substrat.

\subsubsection{La méthode la largeur intégrale : microdéformations et taille des Domaines Cohérents} de Diffraction (DCD).

Ce modèle est basé sur l'idée que le profil du pic de diffraction dans l'espace réciproque résulte de la convolution de trois pics :

i le premier représente l'élargissement instrumental.

ii le second représente l'élargissement dû à la taille des DCD.

iii le troisième représente l'élargissement dû aux microdistorsions du réseau cristallin (quantité de défauts).

La taille des DCD et les microdéformations sont évaluées après déconvolution du profil du pic expérimental en assumant un profil gaussien. Les détails concernant les méthodes de déconvolution sont décrit dans la référence [9].

2.2.1.3 Diffraction aux petits angles : qualité de l'interface et de la périodicité.

Dans le domaine des petits angles, les pics de Bragg sont la résultante de la réflexion des rayons $\mathrm{X}$ sur les interfaces et non sur les plans cristallographiques. Les positions et les profils de ces pics sont donc reliés respectivement à la périodicité et à la qualité des interfaces.

\subsubsection{Spectrométrie d'absorption $\mathrm{X}$}

Afin d'étudier l'ordre local de ces multicouches, des expériences d'absorption X ont été effectuées sur la station EXAFS I au LURE (Orsay-France) de l'anneau D.C.I. fonctionnant à $1,85 \mathrm{GeV}$, aux seuils $L_{\text {in }}$ de l'or et du platine. Pour chacun des spectres, des mesures ont été effectuées avec un temps d'acquisition de $3 \mathrm{sec}$. par pas de $3 \mathrm{eV}$. L'utilisation de la technique de détection des électrons de conversion, grâce à un dispositif fonctionnant à la température de l'azote liquide $(77 \mathrm{~K}) \mathrm{mis}$ au point au laboratoire [10], nous permet d'obtenir des informations sur une faible profondeur dans le matériau étudié (de l'ordre de quelques centaines de $\mathrm{nm}$ ). Les différents paramètres pouvant être déduits d'une telle analyse EXAFS concernent dans le cas présent uniquement l'ordre topologique autour d'un type d'atome donné (suivant le seuil choisi) : nombre de coordinance $\mathrm{N}$, distance des premiers voisins $\mathrm{R}$ et écart quadratique moyen à la position d'équilibre $\sigma_{\mathrm{D}}$.

\section{Résultats et discussion}

Les spectres de diffraction aux grands angles, représentés figure 3 pour un domaine angulaire de 30 à $90^{\circ}$, sont comparables à celui d'une phase cubique face centrée (cfc) unique dont le paramètre de maille serait intermédiaire entre celui de l'or massif $(0,4078 \mathrm{~nm})$ et celui du platine massif $(0.3924 \mathrm{~nm})$. Dans le tableau 2, nous avons reporté les positions angulaires 
(20) mesurées des différentes raies du spectre de ces multicouches numérotées dans l'ordre des raies caractéritiques d'une phases cfc afin de comparer ces résultats à ceux donnés par l'or et le platine massif. La valeur du paramètre de maille calculée par régression linéaire sur les neuf pics de diffraction détectés est approximativement la même pour les deux périodes $\left(a_{5 / 5}=0,3980 \mathrm{~nm}\right.$ et $\left.a_{1 / 212}=0,4006 \mathrm{~nm}\right)$ et correspondrait à une composition équiatomique $\mathrm{Au}_{50} \mathrm{Pt}_{50}$ sur la base d'une loi de Vegard. Des résultats similaires ont été observés par diffraction des électrons en Microscopie Electronique à Transmission (MET) sur des coupes planes pour les deux périodes. On observe par cette méthode de caractérisation une série d'anneau tout à fait cohérente avec une phase cfc unique de paramètre de maille $0.3965 \mathrm{~nm}$. L'intérêt de la diffraction des électrons en MET par rapport à la diffraction des rayons X est de mesurer les distances interréticulaires perpendiculairement à la normale à la surface alors que les rayons $\mathrm{X}$ les mesurent selon cette normale.
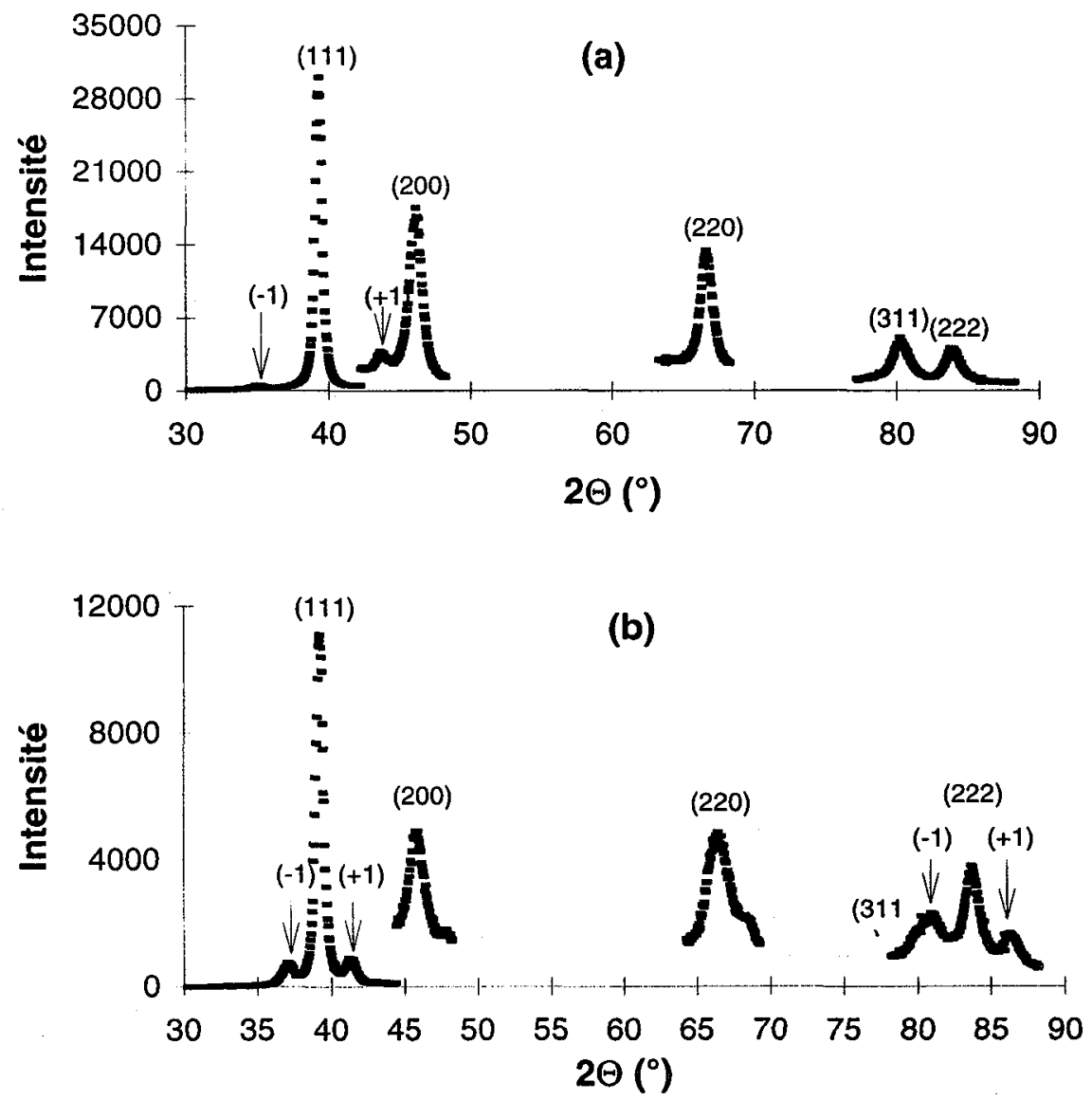

Figure 3: Spectre de diffraction aux grands angles des multicouches $\mathrm{Au} / \mathrm{Pt}$ mesuré avec une longueur d'onde de $0,15406 \mathrm{~nm}$ : (A) période $5 / 5$ et (B) période $12 / 12$ (plans atomiques). Les flèches indiquent les positions des pics satellites autour des réflexions (111) and (222). Les intensités de diffraction pour les raies (200), (220), (311) et (222) ont été multipliées par un coefficient égale à 5,5 et 20 pour les courbes (A) et (B) respectivement. 
Les films minces d'or ou de platine sont généralement texturés : les plans denses (111) sont orientés préférentiellement dans le plan perpendiculaire à la direction de croissance. Les multicouches $\mathrm{Au} / \mathrm{Pt}$ ou $\mathrm{Pt} / \mathrm{Au}$ que nous avons déposées, présentent une texture (111) peu marquée comme le montre le rapport des intensités des pics de diffraction des raies (111) et (200) de la figure 3. Le rapport des intensités des pics de diffraction entre les raies (111) et (200) est plus grand pour la plus grande période $\left(I_{\max (111)} / I_{\max (200)}=44\right)$ que pour la plus petite (10). De plus, les pics de diffraction des raies (400), (331), (420) et (422) sont mieux définis pour la période la plus faible.

La taille des domaines cohérents de diffraction et les microdéformations déduites par la méthode de la largeur intégrale sont de l'ordre de $(10 \pm 1) \mathrm{nm}$ et $(3,5 \pm 0,5) \times 10^{-3}$ respectivement. Ces valeurs ne dépendent pas de la période et correspondent à la direction de croissance du film. La taille des DCD est nettement supérieure à la période la plus grande. Ce point pourrait être corrélé à l'existence d'une cohérence cristallographique partielle aux interfaces entre l'or et le platine.

\begin{tabular}{|c|c|c|c|c|c|c|c|c|c|}
\hline$(\mathrm{hkl})$ & 111 & 200 & 220 & 311 & 222 & 400 & 331 & 420 & 422 \\
\hline $\mathrm{Au}$ & 38,2 & 44,4 & 64,6 & 77,6 & 81,7 & 98,1 & 110,8 & 115,3 & 135,4 \\
\hline $5 / 5$ & 39,1 & 45,7 & 66,5 & 80,1 & 83,9 & 102,0 & 115,1 & 120,2 & 142,6 \\
\hline $12 / 12$ & 39,0 & 45,4 & 66,3 & 79,9 & 83,4 & 101,8 & 114,8 & 119,1 & 139,8 \\
\hline $\mathrm{Pt}$ & 39,7 & 46,2 & 67,4 & 81,2 & 85,7 & 103,5 & 117,7 & 122,8 & 148,2 \\
\hline
\end{tabular}

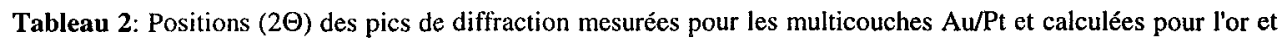
le platine massif. $L^{\prime}$ erreur commise sur la position $(2 \Theta)$ des pics est de $0,1^{\circ}$.

Les pics satellites mis en évidence figure 3 autour des réflexions (hkl) sont corrélés avec la périodicité des multicouches dans leur direction de croissance (normale au plan de surface). Les valeurs de la périodicité déduites de la position de ces pics satellites sont données dans le tableau 3. Ces valeurs sont inférieures à celles données par le cristal piézo-électrique utilisé pour contrôler les épaisseurs déposées.

Comme le montre la figure 4, cette périodicité est aussi visible dans le domaine des petits angles ( autour du faisceau direct) à la même position angulaire $\left(2 \theta=4,12^{\circ}\right.$ ) pour un angle d'incidence $\Omega$ faible (a) ou grand (b). Ces deux pics très larges sont parfaitement homothétiques. Les valeurs de la périodicité déduites de la position de ces pics aux petits angles sont proches de celles trouvées à partir des pics satellites mesurés aux grands angles (voir tableau 3).

\begin{tabular}{|c|c|c|c|}
\hline \multirow{2}{*}{$\begin{array}{c}\text { Epaisseur Bicouche (nm) } \\
\text { (cristal-piézo) }\end{array}$} & \multicolumn{2}{|c|}{ Grands Angles (nm) } & \multirow{2}{*}{ Petits Angles (nm) } \\
\cline { 2 - 3 } & $(111)$ & $(222)$ & \\
\hline 2,4 & 2,25 & $\mathrm{X}$ & 2,15 \\
\hline 5,6 & 4,45 & 4,50 & $\mathrm{X}$ \\
\hline
\end{tabular}

Tableau 3: Valeurs expérimentales de la période relative aux multicouches Au/Pt ou Pt/Au calculées à partir de la position des pics satellites de diffraction aux grands angles et du spectre de diffraction aux petits angles. 


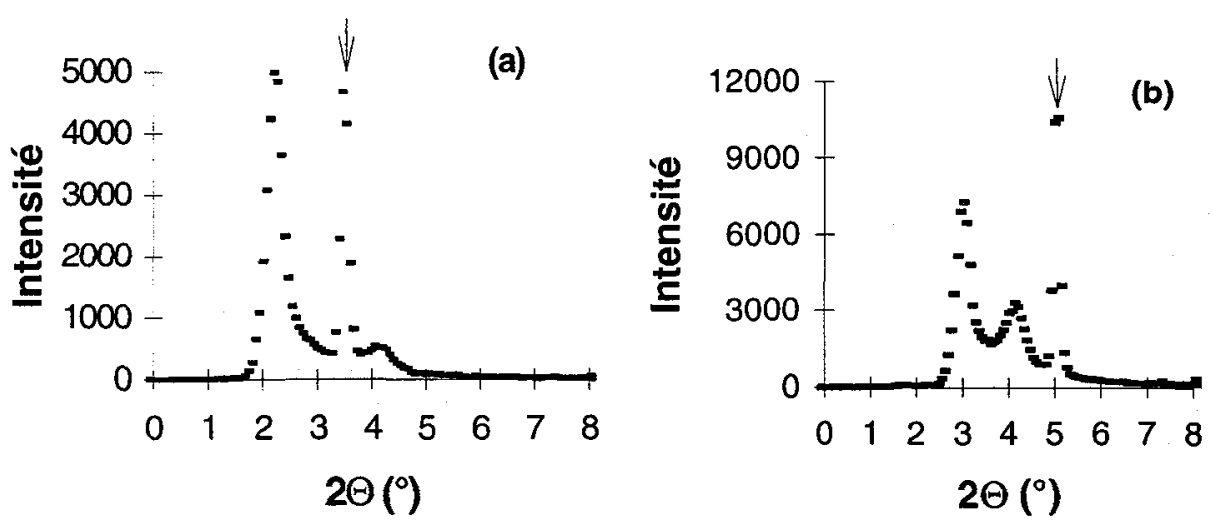

Figure 4: Spectres de diffraction aux petits angles mesurés pour les multicouches $\mathrm{Au} / \mathrm{Pt}$ de période $5 / 5$ plans atomiques avec deux angles $\Omega$ diffèrents et supérieurs aux angles critiques de l'Au et du Pt $\left(0,55\right.$ et $0,58^{\circ}$ respectivement) : a) $1,7^{\circ}$ et b) $2,5^{\circ}$. Les flèches indiquent la position de la réflexion du faisceau direct.

Dans le cas des larges périodes, le signal dans le domaine des petits angles est certainement masqué par le faisceau direct. De plus, notre appareil expérimental n'est pas adapté pour de tel mesure. La largeur des pics aux petits angles est la signature d'une très forte rugosité d'interface due au mode de croissance des multicouches Au/Pt pour d'aussi faibles périodes : formation d'îlots d'or puis de platine (mécanisme de Volmer-Weber) [11].

Les contraintes résiduelles dans des multicouches ont été déterminées d'une part par analyse des spectres de rayons $\mathrm{X}$ et d'autre part selon la méthode de Stoney (courbure du substrat), Nous obtenons des contraintes de même ordre de grandeur par les deux méthodes (600 MPa) mais de signe opposé : en compression par diffraction des rayons $\mathrm{X}$ et en tension par la méthode de Stoney. De plus, les résultats obtenus par ces deux méthodes sont similaires quelle que soit la série considérée. Cette différence peut être attribuée soit à des contraintes de cohérence soit à la méthode de diffraction elle même.

La forme du signal XANES est la signature de la nature des proches voisins. Les Figures 5 a) et b) montrent clairement que les proches voisins d'un atome d'or sont des atomes d'or et que les proches voisins d'un atome de platine sont essentiellement des atomes de platine.
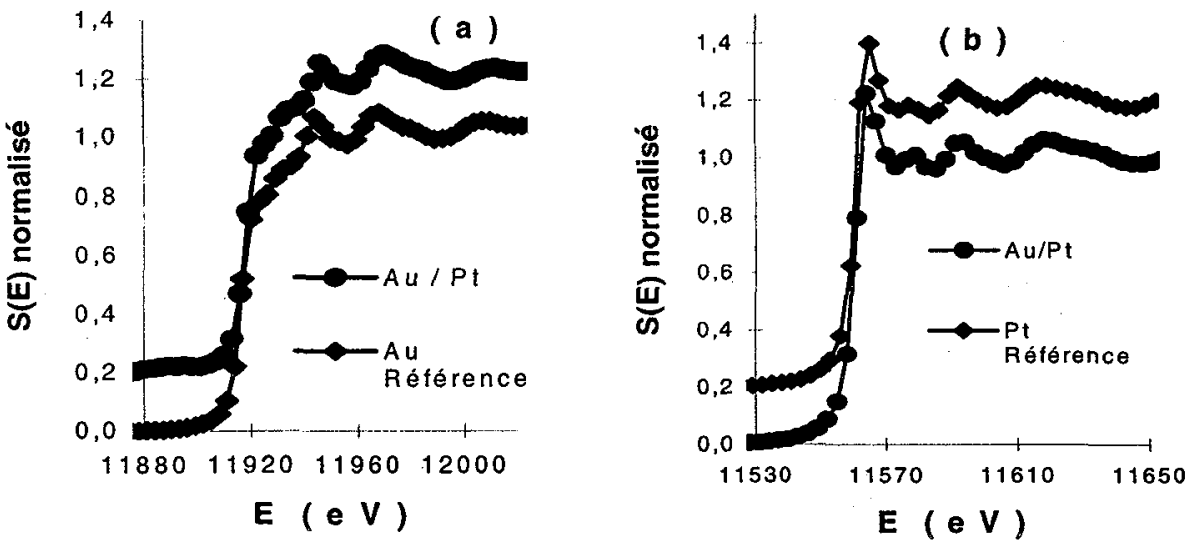

Figure 5 : Spectres XANES au seuil $L_{\text {III }}$ de l'or a) et spectre XANES au seuil $L_{\text {III }}$ du platine b). 
La spectrométrie d'absorption établit clairement que l'or et le platine ne sont pas mélangés mais regroupés en amas d'élément pur.

La principale difficulté de l'étude par spectrométrie d'absorption $\mathrm{X}$ de ce système multicouche $\mathrm{Au} / \mathrm{Pt}$ et $\mathrm{Pt} / \mathrm{Au}$ est liée au fait que les deux seuils sont très proches l'un de l'autre. Ainsi, les oscillations EXAFS du platine sont encore perceptibles au seuil $\mathrm{L}_{\text {lII }}$ de l'or : un dépouillement plus complexe doit être mené. $\mathrm{Vu}$ les très faibles différences entre les amplitudes et phases de retrodiffusion de l'or et du platine [12], il est impossible de déterminer par EXAFS la nature chimique des atomes voisins : les simulations [13] ont donc été menées en ne considérant qu'un seul type d'atome autour de l'atome central, sans changement notable dans la qualité de nos ajustements. Les résultats sont cependant tout à fait fiables, et les incertitudes peuvent être annoncées comme suit : $\Delta N=1, \Delta \sigma_{D}=0,001 \mathrm{~nm}$ et $\Delta R$ $=0,001 \mathrm{~nm}$. Les différents résultats quantitatifs ainsi obtenus sont récapitulés dans le tableau 4. Les paramètres de maille de l'or et du platine se rapprochent d'autant plus que la période est faible, mais ils restent différents. Les nombres de coordinance (voisins de douze) et les faibles valeurs de désordre $\left(\sigma_{D}\right)$ indiquent que les amas d'or et de platine sont de taille importante et relativement bien ordonnés.

\begin{tabular}{|c|c|c|c|c|c|c|c|c|c|}
\hline \multicolumn{4}{|c|}{ seuil de l'or } & \multicolumn{5}{c|}{ seuil du platine } \\
\hline & $\mathrm{N}$ & $\begin{array}{c}\sigma_{\mathrm{D}} \\
(\mathrm{nm})\end{array}$ & $\mathrm{R}(\mathrm{nm})$ & $\mathrm{a}_{0}(\mathrm{~nm})$ & & $\mathrm{N}$ & $\begin{array}{c}\sigma_{\mathrm{D}} \\
(\mathrm{nm})\end{array}$ & $\mathrm{R}(\mathrm{nm})$ & $\mathrm{a}_{0}(\mathrm{~nm})$ \\
\hline $\mathrm{Au}$ massif & 12 & 0,006 & 0,288 & 0,408 & $\mathrm{Pt}$ massif & 12 & 0,006 & 0,277 & 0,392 \\
\hline $\mathrm{Au} / \mathrm{Pt}(5 / 5)$ & 11 & 0,006 & 0,285 & 0,402 & $\mathrm{Au} / \mathrm{Pt}(5 / 5)$ & 12 & 0,007 & 0.281 & 0,397 \\
\hline $\mathrm{Au} / \mathrm{Pt}(12 / 12)$ & 11 & 0,006 & 0,286 & 0,404 & $\mathrm{Au} / \mathrm{Pt}(12 / 12)$ & 12 & 0,007 & 0,279 & 0,395 \\
\hline
\end{tabular}

Tableau 4 : Résultats obtenus par simulation des signaux EXAFS : $N$ le nombre de coordinance, $R$ la distance entre premiers voisins, $\sigma_{D}$ l'écart quadratique moyen à la position d'équilibre, et a le paramètre de maille déduit.

\section{Conclusion}

Dans un papier récent, Michaelsen [4] a clairement démontré les limites de la diffraction des rayons $\mathrm{X}$ pour caractériser des systèmes $\mathrm{Cu} / \mathrm{Co}$ inhomogènes. Ses résultats indiquent que des inhomogènéités cohérentes doivent avoir une taille supérieure à plusieurs nanomètres avant d'être détectées par la diffraction conventionnelle des rayons $\mathrm{X}$. Nos multicouches $\mathrm{Au} / \mathrm{Pt}$ et $\mathrm{Pt} / \mathrm{Au}$ ont une très faible période. Nous sommes donc dans le même cas que Michaelsen, il est impossible de faire la distinction par diffraction des rayons $\mathrm{X}$ entre une solution solide et un système multicouche de faible période. Nos spectres montrent néanmoins que la périodicité $\mathrm{Au} / \mathrm{Pt}$ existe et perturbe l'intensité diffractée quelque soit la géométrie utilisée. La spectrométrie d'absorption $X$, révèle la présence d'or et de platine purs. Le rapprochement des paramètres de maille de l'or et du platine mesurés par EXAFS ne justifie pas la similitude du spectre de diffraction $\mathrm{X}$ aux grands angles avec celui d'une solution solide.

La mesure des contraintes et des microdéformations est toujours possible puisque nous avons des pics de diffraction mais le sens qu'il faut leur donner reste discutable. Les microdéformations ne sont plus seulement la résultante des défauts cristallins; il faut leur ajouter l'effet des défauts de périodicité. Le déplacement mesuré des pics de diffraction est-il caractéristique d'éventuelles contraintes ou des phénomènes de convolution? 
[1] K.F. Badawi, A. Declemy, A. Naudon et Ph. Goudeau, J.Phys.France 2.

[2] K.F. Badawi, Ph. Goudeau, J. Pacaud, C Jaouen et al., Nucl. Inst. and Meth. in Phys. Res. B80181, (1993) 404.

[3] K.F. Badawi, N. Durand, Ph. Goudeau et V. Pelosin, Appl. Phys. Lett. 65 (1994) 30753077.

[4] E. Michaelsen, Phil. Mag. A 72 (1995) 813-828.

[5] Techniques de L'ingénieur -4-1995-V. M1 I p. M70-126.

[6] Colin J. Smithells, Metals Reference Book, M.C., D.sc., A.R.C.S., F.I.M., p. 907.

[7] Ph. Goudeau, K.F. Badawi, A. Naudon, M. Jaulin, N. Durand, L. Bimbault et V. Branger, à paraître dans J.Phys IV.

[8] Stoney G.G., Proc. Roy. Soc. SERA82 (1909) p. 172.

[9] N. Durand, L. Bimbault,K.F. Badawi et Ph. Goudeau, J.Phys III.France 4 (1994) 10251032.

[10] J.Mimault, J.J. Faix, T. Girardeau, M. Jaouen et G. Tourillon, Meas. Sci. Technol. 5 (1994) p 482-489.

[11] M. Volmer, Kinetik der Phasenbildung, Steinkopf, Dresden (1939).

[12] A.G. McKale, G.S. Knapp et S.K. Chan, Phys. Rev. B 33 (1986) 841.

[13] A. Michalowicz, Thèse d'état, Université Paris (1990). 